\title{
Performance study of combined test rig for metro train traction
}

\author{
Xingjian DONG ${ }^{1,2}$, Shengxian ZHUANG ${ }^{1 *}$ \\ 1. School of Electrical Engineering, Southwest Jiaotong University, Chengdu 610031, China \\ 2. Hunan Yinhe Electric Corporation, Changsha 410000, China
}

\begin{abstract}
This paper deals with a combined test rig for a traction system in the laboratory environment. An experimental system was designed and implemented to verify the performance of the traction system for a metro train. For a highly accurate control of the system, a hybrid control algorithm combining vector control and slip frequency control was applied to control the traction inverter. The design method of the flywheels, which represent the equivalent model of the train moment inertia, was elaborated. A train runtime diagnosis system was completed by adopting the multifunction vehicle bus (MVB) protocol. The dynamic performance of the metro power traction system was emulated under the control of the train runtime diagnosis system. Using the combined test rig, the performances of the traction system in traction, braking, temperature rise, etc., were verified through traction and breaking experiments.
\end{abstract}

Key words: traction system; variable voltage variable frequency inverter; flywheels; combined test

(C) 2011 JMT. All rights reserved.

\section{Introduction}

$\mathrm{T}$ he power traction system is an important part of the metro system. With the development of power electronic technology in the railway traffic domain, the metro power traction system has been modified from the traditional direct current (DC) drive mode into alternating current $(\mathrm{AC})$ drive mode [1-3]. The development of high power level AC drive system is often verified using a combined test system that is composed of a traction inverter, several traction motors and a vehicle control system.

Traction system reliability is the most important factor of train safety operation [4]. Traction and braking performance are not only influenced by the inverter, but the traction motors, transmission system, braking system, and signal system as well [5]. In most cases, performance test under software simulation results in long development cycle and high cost. It is essential to establish a combined test system including vehicle, motors, signals, and communication network for traction system performance test before actual operation.

A reciprocal power-fed $\mathrm{AC}$ drive test rig was proposed in [6], where an AC motor was used to simulate the vehicle. The system could simulate the traction and braking charac-

Received June 28, 2011; revision accepted July 23; 2011

*Corresponding author. Tel.: +86-28-87601933; E-mail: zhuangsx@sina.com (S.X. ZHUANG)

(C) 2011 JMT. All rights reserved doi: 10.3969/j.issn.2095-087X.2011.03.003 teristics at some fixed power points, but could not simulate the inertia characteristics of the vehicle continuously.

This paper deals with the design and development of a megawatt (MW) level combined test rig to complete the dynamic characteristic test for a metro power traction system in the laboratory environment. In this test rig, the configuration and control of electrical parts is consistent with the actual vehicle: equivalent flywheels are adopted to simulate the inertia of vehicle, and the multi-function vehicle bus (MVB) [7] communication protocol is adopted to form the train runtime diagnosis system. Simulation of the traction and braking characteristics of the traction system for the metro are evaluated according to standard IEC61377-1 [8]. With the proposed combined test rig, the dynamic performance of the tested metro power traction system can be evaluated continuously in full speed range.

Fig. 1 shows the vehicle communication interface of a metro train, where abbreviations are defined as follows:
APU Auxiliary power unit
CCU Central control unit
DU Display unit
MVB_IOS Multi-function vehicle bus in/out system
$\mathrm{M} i \quad$ Motor train unit $i(i=1,2,3,4)$
TC Train communication network
VATC A Vehicle carbone controller A
VATC B Vehicle carbone controller B
VCU $\quad$ Vehicle control unit
VVVF Variable voltage variable frequency 
The main goals of the experimental test rig are as follows:

- Verification of the traction and breaking performance.

- Verification of the temperature rise performance.

- Troubleshooting of the traction system interface with safety.

\section{System configuration}

The proposed experimental system consists of four traction motors controlled in parallel by one VVVF inverter with breaking chopper. Two gear boxes are connected to two flywheels respectively. The scheme of the experimental system is shown in Fig. 2.

\section{Unit implementation of the proposed experimental system}

\subsection{Rectifier bank}

The rectifier bank which provides a DC voltage bus for the VVVF inverter consists of three same type 12 pulses diode rectifiers cascaded with an adjustable 12 pulses diode rectifier, where each fixed diode rectifier outputs $600 \mathrm{~V}$, and the adjustable diode rectifier varies from 0 to $600 \mathrm{~V}$. The structure diagram of the rectifier bank is shown in Fig. 3.

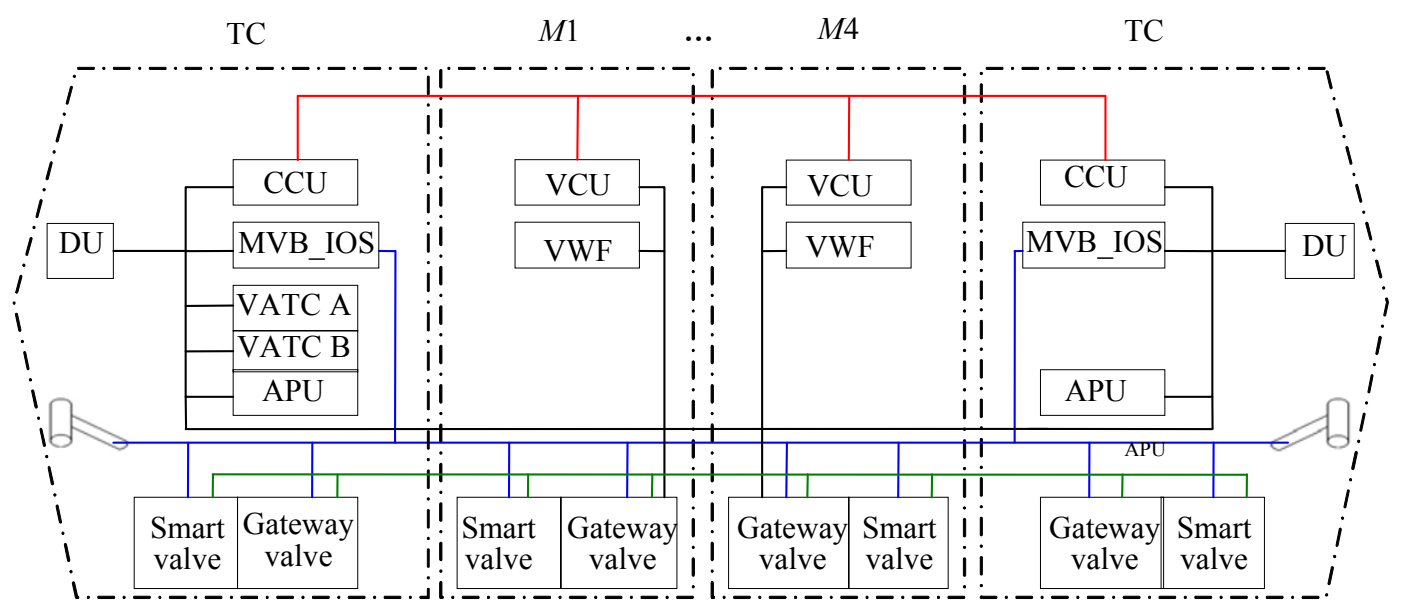

Fig. 1 Communication interface of metro train

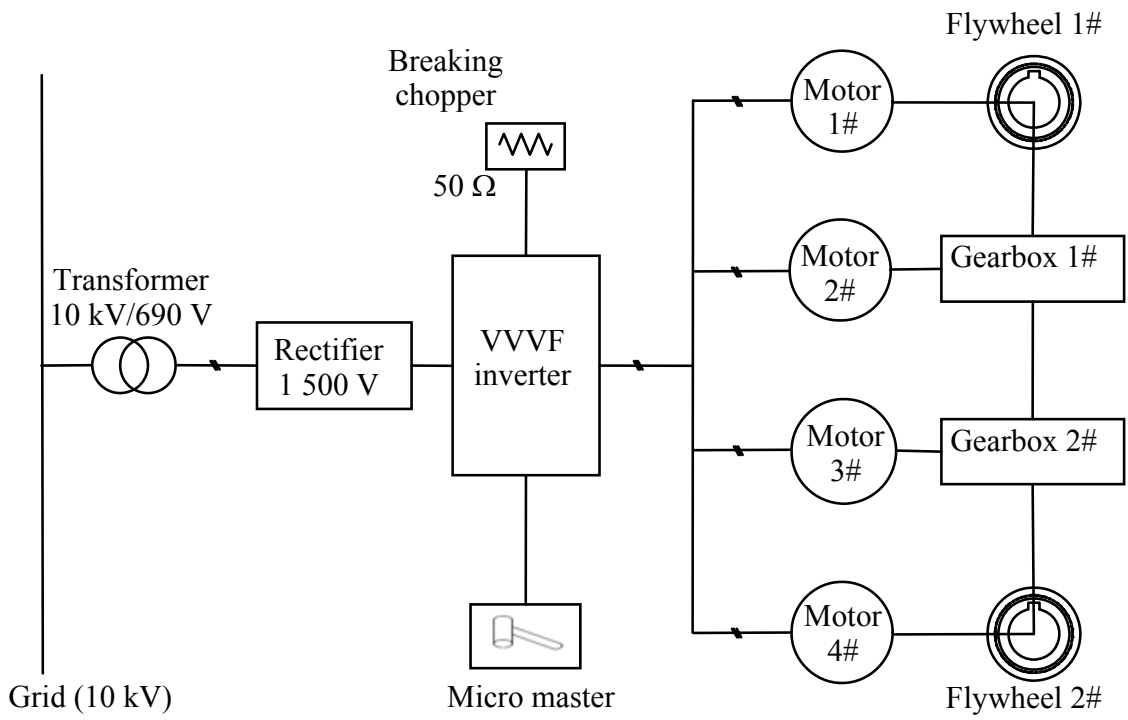

Fig. 2 Scheme of power traction simulator system 


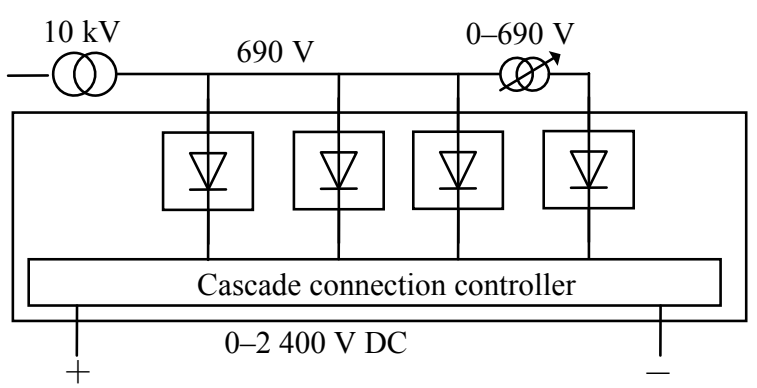

Fig. 3 Structure diagram of rectifier bank

\subsection{VVVF traction inverter}

A 1 MW slip vector control inverter is adopted as the VVVF traction inverter in the proposed system. The inverter of DC link voltage ranging from $1500 \mathrm{~V}$ to $1900 \mathrm{~V}$ follows a constant torque control below the rated speed and a constant power control above the rated speed. The breaking chopper unit keeps a constant DC link voltage when the DC link voltage exceeds $1900 \mathrm{~V}$.

\subsubsection{Operation characteristic}

Fig. 4 shows the operation characteristic of the traction system. In region $C$, the constant slip frequency control is applied. The output voltage is kept constant, and torque is generated by increasing the output frequency. In region $D$, the traction system runs under an inertial mode. Constant power braking is active in region $E$, with constant torque braking in region $F$.

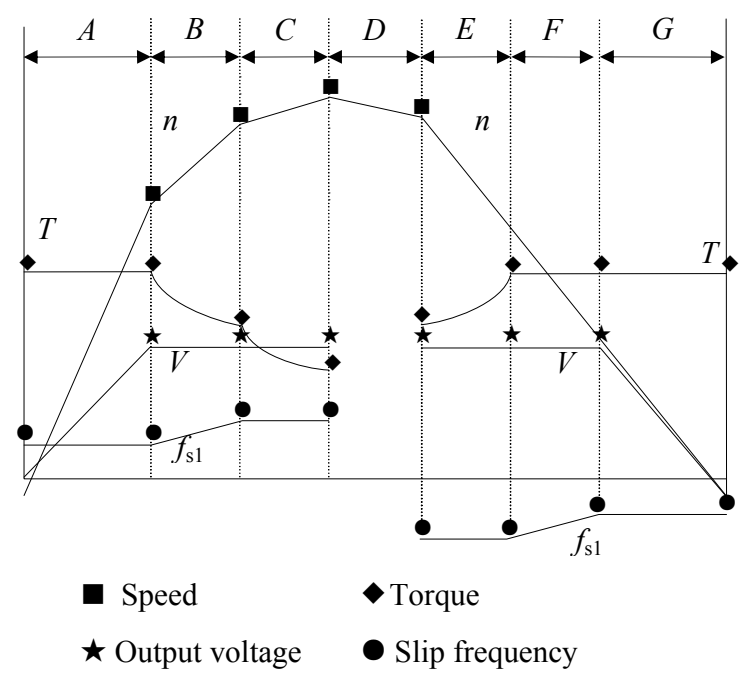

Fig. 4 Operation characteristic of traction system

\subsubsection{Control algorithm}

For highly accurate control, a hybrid control algorithm combining vector control and slip frequency con- trol is applied and shown in Fig. 5. In the low frequency region, vector control is applied to achieve a high dynamic performance. In the high frequency region and over modulation region, slip frequency control is applied by controlling the average torque and current.

\subsection{Designation of flywheels}

Flywheels represent the moment inertia of a vehicle with weight of $64 \mathrm{t}$, wheel diameter of $840 \mathrm{~mm}$, and maximum speed of $120 \mathrm{~km} / \mathrm{h}$. Fig. 6 shows the structure of a flywheel. A view of the flywheels is shown in Fig. 7.

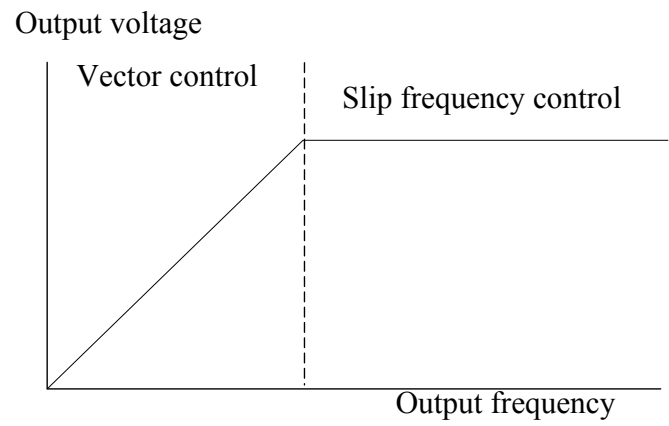

Fig. 5 Hybrid control of traction inverter

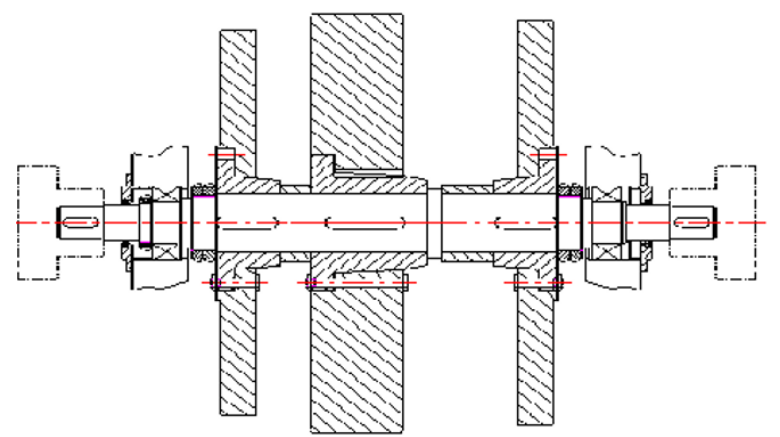

Fig. 6 Structure of a flywheel

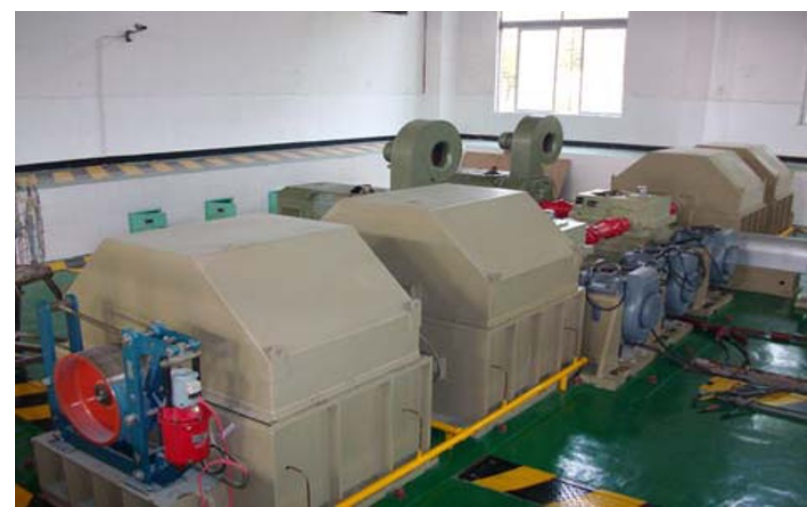

Fig. 7 View of actual flywheels 
According to the principle of momentum balance [9], one has

$$
\begin{aligned}
& \frac{1}{2} M_{\mathrm{vhc}} V_{\mathrm{vhc}}^{2}+\frac{1}{2} J_{\mathrm{vhc}} \omega_{\mathrm{vhc}}^{2}=\frac{1}{2} J_{\mathrm{flw}} \omega_{\mathrm{flw}}^{2}, \\
& \omega_{\mathrm{vhc}}=\frac{2 \pi n_{\mathrm{mot}}}{\eta_{\mathrm{vhc}}} \\
& \omega_{\mathrm{flw}}=\frac{2 \pi n_{\mathrm{mot}}}{\eta_{\mathrm{flw}}}
\end{aligned}
$$

where $M_{\mathrm{vhc}}$ is the mass of vehicle, $V_{\mathrm{vhc}}$ is the runtime velocity of vehicle, $J_{\mathrm{vhc}}$ is the moment inertia of vehicle wheels, $\omega_{\text {vhc }}$ is the angular velocity of vehicle wheels, $J_{\text {flw }}$ is the equivalent moment inertia of flywheels, $\omega_{\text {flw }}$ is the angular velocity of flywheels, $n_{\text {mot }}$ is the motor speed of traction motors, $\eta_{\mathrm{vhc}}$ is the transmission ratio of the vehicle, and $\eta_{\mathrm{flw}}$ is the transmission ratio of the flywheels.

From Eq. (1) and Eq. (2), the equivalent moment inertia of the flywheels can be written as follows:

$$
J_{\text {flw }}=\eta_{\text {flw }} \frac{\eta_{\mathrm{vhc}}^{2} M_{\mathrm{vhc}} V_{\mathrm{vhc}}^{2}+4 \pi^{2} J_{\mathrm{vhc}} n_{\mathrm{mot}}^{2}}{4 \pi^{2} \eta_{\mathrm{vhc}}^{2} n_{\mathrm{mot}}^{2}} .
$$

Parameters of vehicle and flywheels in the proposed system are shown in Table 1.
Table 1 Parameters of vehicle and flywheels

\begin{tabular}{cc}
\hline Parameter & Value \\
\hline$M_{\text {vhc }}$ & $64 \mathrm{t}$ \\
$V_{\text {vhc }}$ & $80 \mathrm{~km} / \mathrm{h}$ \\
$J_{\text {vhc }}$ & $460 \mathrm{~kg} \cdot \mathrm{m}^{2}$ \\
$n_{\text {mot }}$ & $2200 \mathrm{rpm}$ \\
$\eta_{\text {vhc }}$ & 6.31 \\
$\eta_{\text {flw }}$ & 4 \\
\hline
\end{tabular}

\section{Experimental results}

\subsection{Verification of traction and breaking characteristic}

The master controller is put on position 'P4' when the traction system starts, meaning that full torque is output. After $30 \mathrm{~s}$ acceleration, the master controller is put on position 'P0' and lasts for $15 \mathrm{~s}$, and the system runs under the inertial mode. Finally, a $15 \mathrm{~s}$ braking is done by putting the master controller on position 'B4'.

Full power traction characteristic and braking characteristic of the traction system are shown in Fig. 8, where $N$ is vehicle speed, in $\mathrm{km} / \mathrm{h} ; P_{1}$ is the root mean square (RMS) value of input power, in $\mathrm{kW} ; T$ is mechanical torque, in $\mathrm{N} \cdot \mathrm{m} ; V_{\text {ac }}$ is the RMS value of the line to line AC voltage, in $\mathrm{V}$; and, $V_{\mathrm{dc}}$ is the voltage of the DC bus, in $\mathrm{V}$.

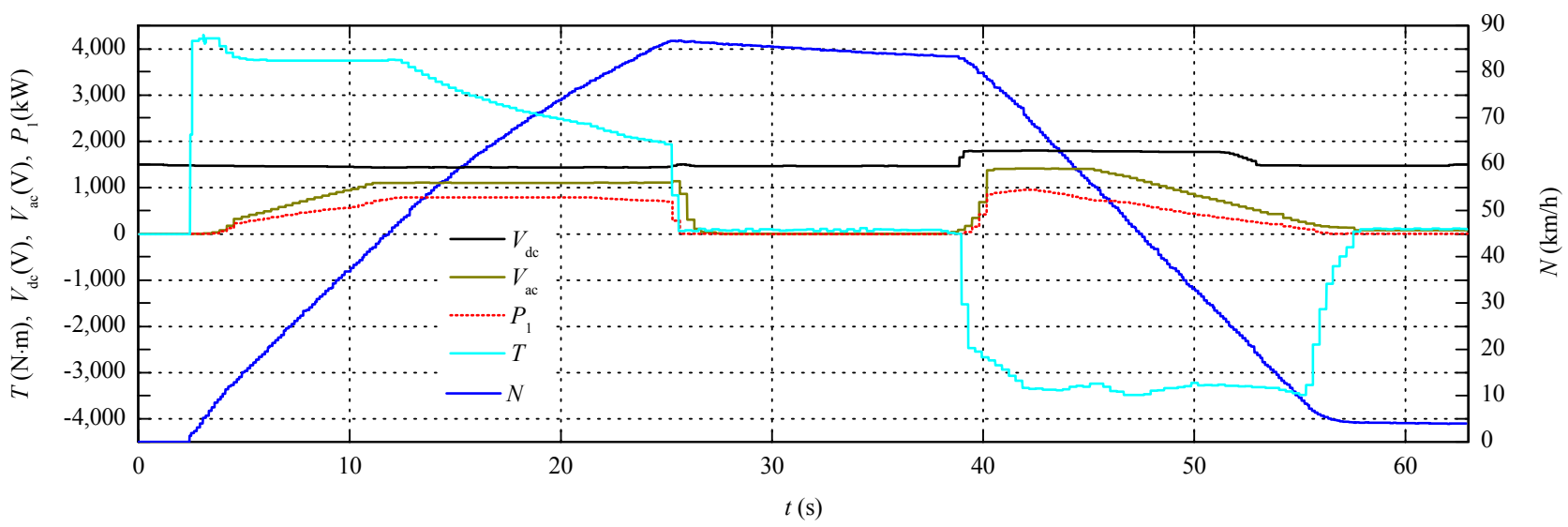

Fig. 8 Full power traction characteristic and braking characteristic of the traction system

In the traction condition, constant torque control is applied when the speed is less than $44 \mathrm{~km} / \mathrm{h}$ while constant power control is applied when the speed is over $44 \mathrm{~km} / \mathrm{h}$.

In the constant speed condition, VVVF inverter stops the power supply.

In the braking condition, the simulator system brakes with a constant torque, the DC bus voltage increases and remains at a constant value after the braking resistor is switched on.

\subsection{Verification of temperature rise characteristic}

To simulate the temperature rise characteristic of the traction system, 60 cycles of power and braking operation is done in $1 \mathrm{~h}$, where one cycle time is $1 \mathrm{~min}$ (traction: $30 \mathrm{~s}$, stopping supply: $15 \mathrm{~s}$, braking: $15 \mathrm{~s}$ ).

Fig. 9 shows a 5 min temperature rise characteristic of the traction system. 


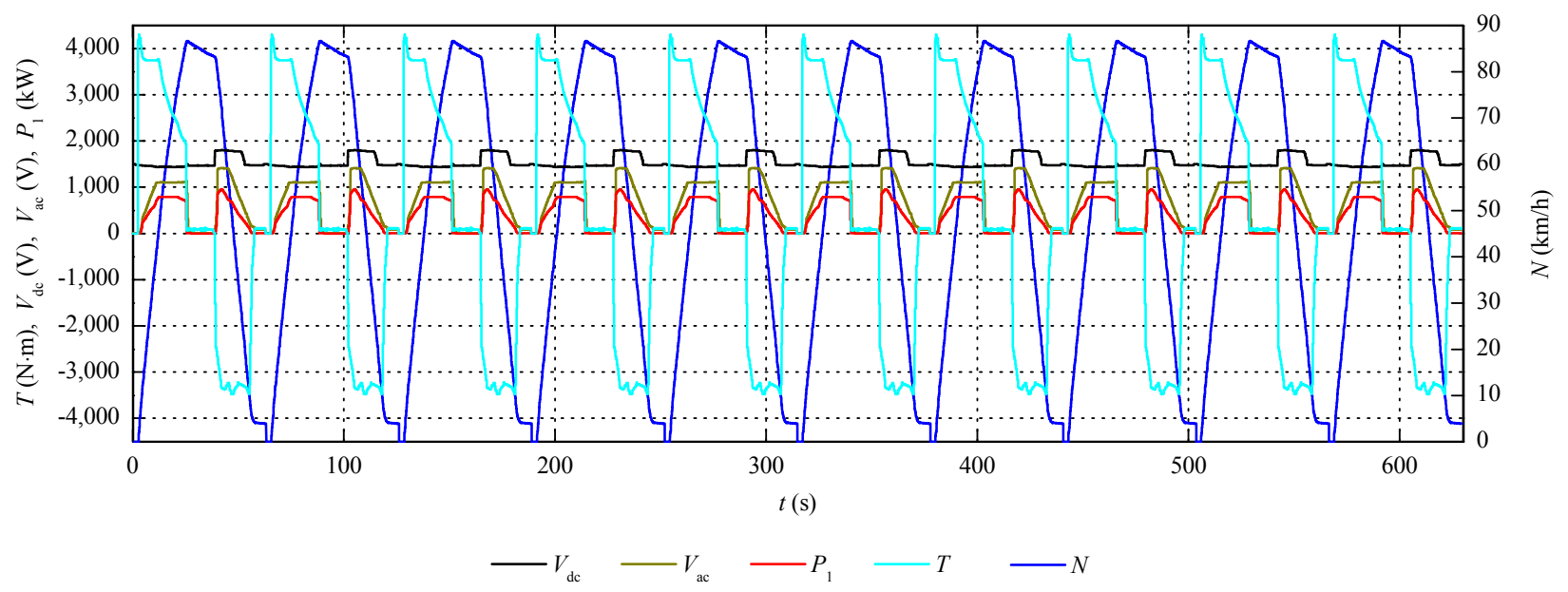

Fig. 9 Temperature rise characteristic of the traction system

\section{Discussion}

It can be seen from Fig. 8 that the system starts with a higher torque due to the voltage drop across the motor resistor at low frequency. When the inverter stops the power supply, the speed decreases according to the inertia characteristics of the vehicle. The system performance is consistent with the designed operation characteristics of the traction system shown in Fig. 4.

\section{Conclusion}

This paper deals with the design and implementation of an experimental system to demonstrate the combined characteristics of the metro power traction system. The flywheels are adopted to simulate the inertia of vehicle. A hybrid control algorithm combining vector control under nominal frequency and slip frequency control above nominal frequency is applied to control the traction inverter, and useful combined test results are completed via MVB interface according to standard IEC61377-1.

The following characteristics are verified experimentally on the combined test rig:

- Verification of the traction and breaking performance.

- Verification of the temperature rise performance.

- Troubleshooting of the traction system interface with safety.

\section{Acknowledgements}

This research was supported by the Innovation Funds for Technology Based Firms (09C26214301971).

\section{References}

[1] S. Sone, Power electronic technologies for low cost and conservation on world railways vehicles, In: Int. Conf. on Power Electronics, Tokyo, 2000: 452-458.

[2] Q.L. Zheng, F. Lin, Modern rail transportation and the reciprocal power-fed AC drive experiment system, Transaction of China Electron Technical Society, 2005, 20(1): 21-25 (in Chinese).

[3] L.J. Diao, Z.G. Liu, M.S. Shen, et al., Survey of AC drive test system research, Electrical Drive, 2007, 37(9): 3-7 (in Chinese).

[4] S. Sagareli, Traction power systems reliability concepts, In: Proc. of the 2004 ASME/IEEE Joint Rail Conference, Maryland, 2004: 35- 39.

[5] T.M. Jahns, V. Blasko, Recent advances in power electronics technology for industrial and traction machine drives, Proc. of the IEEE, 2001, 89(6): 963-975.

[6] Q.Z. Zhang, Y.H. Deng, Y.M. Pan, Research on improved slip frequency control system based on the reciprocal power-fed AC drive test rig, Power Electronics, 2007, 41(9): 69-71 (in Chinese).

[7] Q. L. Liu, J.J. Guo, W. Wei, Design and implementation of MVB protocol analyzer, In: Proc. of 8th WCICA, Jinan, China, July 7-9, 2010: 5404-5408.

[8] IEC 61377-1, Railway applications-rolling stock-part 1: combined testing of inverter-fed alternating current motors and their control system.

[9] B.S. Xiong, G.C. Cui, C.Q. Zhang, Several methods of calculating the flywheel inertia, Journal of Zhengzhou Industrial Institute, 1995, 16(2): 35-40 (in Chinese).

(Editor: Junsi LAN) 